\title{
Simplified analysis of asymmetric structures with supplemental damping
}

\author{
Rakesh K. Goel ${ }^{* \dagger}$ \\ Department of Civil and Environmental Engineering, California Polytechnic State University, \\ San Luis Obispo, California 93407, U.S.A.
}

\begin{abstract}
SUMMARY
This study investigated the effects of neglecting off-diagonal terms of the transformed damping matrix on the seismic response of non-proportionally damped asymmetric-plan systems with the specific aim of identifying the range of system parameters for which this simplification can be used without introducing significant errors in the response. For this purpose, a procedure is presented in which modal damping ratios computed by neglecting off-diagonal terms of the transformed damping matrix are used in the traditional modal analysis. The effects of the simplification are evaluated first by comparing the aforementioned modal damping ratios with the apparent damping ratios obtained from the complex-valued eigenanalysis. The variation of a parameter that was defined by Warburton and Soni as an indicator of the errors introduced by the simplification is examined next. Finally, edge deformations obtained from the simplified procedure are compared with those obtained from the direct integration of the equations of motion. It is found that the simplified procedure may be used without introducing significant errors in response for most practical values of the system parameters. Furthermore, estimates of the edge deformations, in general, tend to be on the conservative side. Copyright (c) 2001 John Wiley \& Sons, Ltd.
\end{abstract}

KEY WORDS: asymmetric buildings; earthquake response; passive control; plan asymmetry; protective systems; simplified analysis; seismic response; supplemental damping; torsion; viscous damping

\section{INTRODUCTION}

In dynamic analysis of linear systems subjected to earthquake ground motion, it is common to assume that the system is classically or proportionally damped. For such systems, the equations of motion can be transformed into a set of independent modal equations using

\footnotetext{
* Correspondence to: Rakesh K. Goel, Department of Civil and Environmental Engineering, California Polytechnic State University, San Luis Obispo, California 93407, U.S.A.

$\dagger$ E-mail: rgoel@calpoly.edu
}

Contract/grant sponsor: National Science Foundation; contract/grant number: CMS-9812414 
real-valued mode shapes and frequencies. The system response can then be evaluated using standard modal analysis techniques $[1,2]$.

In most systems with supplemental damping devices, however, the assumption of classical damping may not be valid. Such systems are denoted as non-classically or non-proportionally damped. In the past, several different methods for analysis of non-classically damped systems have been proposed. However, most of these methods have limitations as apparent from the following review of the literature on this subject.

An obvious method is to integrate directly the coupled equations of motion [3]. However, this method is numerically inefficient for systems with a large number of degrees of freedom. Mode superposition method, in which only a limited number of important modes are considered, offers an attractive alternative to the direct integration method. For non-classically damped systems, however, the mode superposition method involves complex-valued mode shapes and frequencies [1,3-6]. Further limitations of this method are doubling of the size of the eigenvalue problem and difficulties associated with the use of complex numbers in dynamics response analysis.

In order to overcome the limitations of the complex-valued modal analysis of non-proportional system, hybrid time-domain, frequency-domain procedure has been developed [7-9]. This approach involves solving the coupled modal equations (obtained by using undamped mode shapes and frequencies) iteratively in time domain; only equations corresponding to modes expected to contribute significantly to the total response are considered. Although appealing, this procedure requires solutions of the response in the time-domain, which can be numerically inefficient, and cannot be implemented on most commercially available structural analysis programs. Spectral method for random vibration analysis has also been proposed [10].

A common approach adopted for analysing non-classically damped systems is to diagonalize the transformed damping matrix; the transformation process is described later in this paper. Although several different approaches have been proposed for this purpose, e.g. Reference [11], the most common approach is to simply neglect the off-diagonal elements in the transformed damping matrix [12-14]. The modal damping ratios computed from the diagonal terms of the transformed damping matrix are then used in the modal equations that are uncoupled by mode shapes of the undamped system. The recently developed FEMA guidelines for seismic rehabilitation of buildings $[15,16]$ have adopted this approach for seismic analysis of buildings with supplemental damping devices; the simplified equations for calculating the effective modal damping ratios given in these documents are derived from diagonal terms of the transformed damping matrix while neglecting the off-diagonal terms. This method is appealing to the design professionals because it enables use of the traditional modal analysis methods, including response spectrum analysis, for non-classically damped systems.

The simplification associated with neglecting off-diagonal terms in the transformed damping matrix obviously introduces errors in the response. Warburton and Soni [12] examined errors due to such simplification. For this purpose, a parameter was defined to indicate the level of error in the response. It was found that if this parameter is less than 5 per cent, the errors in response caused by this simplification are no more than 10 per cent.

Most previous studies on seismic analysis of systems with non-proportional damping have been focused on symmetric-plan or planar systems. Some recent studies, e.g. References [6, 17] have begun to examine the seismic behaviour of asymmetric-plan systems with supplemental dampers. Such systems generally belong to the class of systems with non-proportional damping. The controlling parameters of asymmetric-plan systems with supplemental damping have been 
identified and their effects on seismic response evaluated [17]. The analytical methods that were used are either the direct integration of equations of motion [17] or the complex-valued modal analysis [6]. As noted previously, such methods are likely to find limited application in the engineering design practice due to significant computational efforts or due to complexities in implementation.

Therefore, the main objective of this study is to investigate the effects of neglecting offdiagonal terms of the transformed damping matrix on the seismic response of asymmetric systems with supplemental damping. With this objective, a simplified procedure is presented in which modal damping ratios computed by neglecting off-diagonal terms of the transformed damping matrix are used in the modal equations that are uncoupled by mode shapes of the undamped system. An additional objective is to identify the range of system parameters for which the simplified procedure can be used without introducing significant errors in the response.

For this purpose, damping ratios are computed from the simplified procedure and compared with apparent damping ratios obtained from the complex-valued eigenanalysis. The variation of the parameter defined by Warburton and Soni [12] is examined next for a wide range of system parameters. Finally, the edge deformations obtained from the simplified procedure are compared with those obtained from the direct integration of the equations of motion to assess errors introduced by the simplification. It is found that the simplified procedure may be used without introducing significant errors in response for most practical values of the system parameters. Furthermore, estimates of the edge deformations, in general, tend to be on the conservative side.

\section{THEORETICAL BACKGROUND}

Equations of motion for a multi-degree-of-freedom system subjected to ground motion are:

$$
\mathbf{M u ̈}(t)+\mathbf{C u}(t)+\mathbf{K u}(t)=-\mathbf{M r} \ddot{u}_{\mathrm{g}}(t)
$$

in which matrices $\mathbf{M}, \mathbf{C}$, and $\mathbf{K}$ characterize the mass, damping, and stiffness related to the deformations $\mathbf{u}(t)$ at various degrees of freedom, $\mathbf{r}$ is the influence vector, and $\ddot{u}_{\mathrm{g}}(t)$ is the ground acceleration. For a system with $\mathrm{N}$ degrees of freedom (DOF), M, $\mathbf{C}$, and $\mathbf{K}$ are $N \times N$ matrices, and $\mathbf{u}(t)$ and $\mathbf{r}$ are $N \times 1$ vectors. Reviewed briefly next are the modal analysis methods used for proportional and non-proportional systems for solving the equations of motion. This material is included because it provides the basis for the simplified procedure for analysis of asymmetric-plan systems with supplemental damping.

\section{Modal analysis of proportional systems}

Using standard modal decomposition method [2], the coupled $N \times N$ system of second-order differential equations represented by Equation (1) may be transformed to a set of $N$ uncoupled second-order differential equations:

$$
\ddot{q}_{n}(t)+2 \omega_{n}{ }_{n} \dot{q}_{n}(t)+\omega_{n}^{2} q_{n}(t)=\Gamma_{n} \ddot{u}_{\mathrm{g}}(t) ; \quad n=1 \text { to } N
$$


in which $q_{n}(t)$ is the $n$th modal co-ordinate, $\omega_{n}$ and ${ }_{n}$ are frequencies and damping ratio in the $n$th mode of vibration, and $\Gamma_{n}$ is the modal participation factor defined by

$$
\Gamma_{n}=\frac{{ }_{n}^{\mathrm{T}} \mathbf{M r}}{{ }_{n}^{\mathrm{T}} \mathbf{M}{ }_{n}}
$$

where $\quad n$ is the $n$th mode shape of system. The mode shapes and frequencies that are used in Equations (2) and (3) are obtained from the eigenvalue problem for the undamped multidegree-of-freedom system

$$
\left(\mathbf{K}-\omega^{2} \mathbf{M}\right) \Phi=0
$$

which gives $N$ values of $\omega_{n}$ and ${ }_{n}$. Since both $\mathbf{M}$ and $\mathbf{K}$ in structural engineering applications are symmetric and positive-definite, both $\omega_{n}$ and ${ }_{n}$ are real valued. The uncoupling of equations of motion represented by Equation (2) is possible only when the damping matrix C satisfies the following condition:

$$
{ }_{n}^{\mathrm{T}} \mathbf{C}{ }_{r}=0 \text { for } n=r
$$

which implies zero off-diagonal terms in the matrix product $\Phi^{\mathrm{T}} \mathbf{C} \Phi$ ( $\Phi$ is the matrix of all mode shapes of an undamped system); $\Phi^{\mathrm{T}} \mathbf{C} \Phi$ is denoted as the transformed damping matrix in rest of this paper. The condition represented by Equation (5) implies that the damping matrix is orthogonal (or proportional) to the undamped mode shapes of the system. If this condition is not satisfied, the damping is called non-proportional (or non-classical) and the simplification of Equation (2) cannot be achieved.

For proportional systems, $n$ to be used in Equation (2) is calculated from

$$
{ }_{n}=\frac{{ }_{n}^{\mathrm{T}} \mathbf{C}{ }_{n}}{2 \omega_{n}{ }_{n}^{\mathrm{T}} \mathbf{M}{ }_{n}}
$$

and the total response computed from

$$
\mathbf{u}(t)={ }_{n=1}^{N}{ }_{n} q_{n}(t)
$$

\section{Modal analysis of non-proportional systems}

The modal analysis of system with non-proportional damping can be implemented using an alternative state-space formulation [6]:

$$
\mathbf{A} \dot{\mathbf{z}}(t)+\mathbf{B z}(t)=\mathbf{R} \ddot{u}_{\mathrm{g}}(t)
$$

where $\mathbf{z}(t)=\mathbf{u}(t) \dot{\mathbf{u}}(t)^{\mathrm{T}}$ is a $2 N \times 1$ vector, $\mathbf{A}$ and $\mathbf{B}$ are the $2 N \times 2 N$ parameter matrices for the system given by

$$
\mathbf{A}=\begin{array}{cc}
-\mathbf{K} & \mathbf{0} \\
\mathbf{0} & \mathbf{M}
\end{array} \text { and } \mathbf{B}=\begin{array}{ll}
\mathbf{0} & \mathbf{K} \\
\mathbf{K} & \mathbf{C}
\end{array}
$$

and $\mathbf{R}$ is a $2 N \times 1$ vector defined as

$$
\mathbf{R}=\begin{gathered}
\mathbf{0} \\
-\mathbf{M r}
\end{gathered}
$$


For systems with non-proportional damping matrix, mode shapes may be obtained from

$$
(\mathbf{B}+\mathbf{A}) \Theta=0
$$

which leads to $2 N$ complex-valued eigenvalues ${ }_{n}^{*}$ and eigenvectors ${ }_{n}^{*}$. The complex eigenvalues ${ }_{n}^{*}$ appear in complex conjugate pairs in the form of

$$
{ }_{n}^{*}=-{ }_{n}^{*} \omega_{n}^{*}-\mathrm{j} \omega_{n}^{*} \overline{1-{ }_{n}^{* 2}} \text { and } \quad \tilde{\sim}_{n}^{*}=-{ }_{n}^{*} \omega_{n}^{*}+\mathrm{j} \omega_{n}^{*} \overline{1-{ }_{n}^{* 2}}
$$

in which $\omega_{n}^{*}$ and ${ }_{n}^{*}$ are the apparent natural vibration frequency and apparent modal damping ratio, respectively, associated with the $n$th modal pair. Equation (12) may be utilized to obtain the apparent vibration frequencies and apparent modal damping ratios as

$$
\omega_{n}^{*}=\overline{\operatorname{Re}\left(\begin{array}{c}
* \\
n
\end{array}\right)^{2}+\operatorname{Im}\left(\begin{array}{c}
* \\
n
\end{array}\right)^{2}} \text { and } \quad{ }_{n}^{*}=\frac{-\operatorname{Re}\left(\begin{array}{c}
* \\
n
\end{array}\right)}{\overline{\operatorname{Re}\left(\begin{array}{c}
* \\
n
\end{array}\right)^{2}+\operatorname{Im}\left(\begin{array}{c}
* \\
n
\end{array}\right)^{2}}}
$$

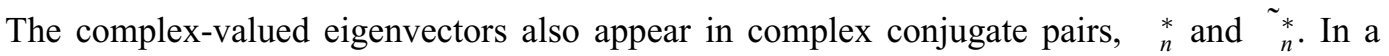
complex-valued eigenvector, each element describes the relative magnitude and phase of the motion of the DOF associated with that element when the system is excited at that mode only. In general, the relative position of each DOF can be out of phase by the amount indicated by the complex part of the mode shape element; all DOF vibrate with the same phase angle if the mode shape is real-valued.

Using complex-valued eigenvectors, $\underset{n}{*}$, equations of motions of Equation (8) can be converted to the following $2 \mathrm{~N}$ uncoupled, first-order differential equations:

$$
\dot{q}_{n}^{*}(t)+{ }_{n} q_{n}^{*}(t)=\Gamma_{n}^{*} \ddot{u}_{\mathrm{g}}(t), \quad n=1 \text { to } 2 N
$$

where

$$
\Gamma_{n}^{*}=\frac{{ }_{n}^{* \mathrm{~T}} \mathbf{R}}{{ }_{n}^{* \mathrm{~T}} \mathbf{A}{ }_{n}^{*}}
$$

is the complex-values modal participation factor. The total response can then be obtained from

$$
\mathbf{u}(t)={ }_{n=1}^{2 n}{ }_{n}^{*} q_{n}^{*}(t)
$$

Note that for a non-proportional system, the following product of mode shape (or eigenvector) matrix, $\Phi$, obtained from eigenanalysis of undamped system (Equation (4)) and the damping matrix, $\mathbf{C}$ is not a diagonal matrix,

$$
\Phi^{\mathrm{T}} \mathbf{C} \Phi=\left[\begin{array}{cccc}
c_{11} & c_{12} & \cdots & c_{1 N} \\
c_{21} & c_{22} & \cdots & c_{2 N} \\
\vdots & \vdots & \ddots & \vdots \\
c_{N 1} & c_{N 2} & \cdots & c_{N N}
\end{array}\right]
$$

that is, the off-diagonal terms $c_{i j, i=j}$ are not necessarily zero. 


\section{SIMPLIFIED MODAL ANALYSIS OF NON-PROPORTIONAL SYSTEMS}

The key to implementation of the simplified modal analysis of non-proportional systems is calculation of the modal damping ratios. These damping ratios can be computed by the following step-by-step procedure:

1. Calculate the mode shapes and frequencies from eigenanalysis of the undamped system (Equation (4)).

2. Assemble the system, damping matrix, $\mathbf{C}$.

$$
\mathbf{C}=\mathbf{C}_{n}+\mathbf{C}_{\mathrm{sd}}
$$

where $\mathbf{C}_{n}$ is a proportional damping matrix of the system without supplemental dampers defined as

$$
\mathbf{C}_{n}=a_{0} \mathbf{M}+a_{1} \mathbf{K}
$$

in which constants $a_{0}$ and $a_{1}$ depend on damping ratios in the selected two undamped vibration modes of the system, and $C_{\mathrm{sd}}$ is the damping matrix due to supplemental dampers which depends on the damping constant and locations of the dampers.

3. Calculate the damping ratio in the $n$th mode from

$$
{ }_{n}=\frac{{ }_{n}^{\mathrm{T}} \mathbf{C}}{2 \omega_{n}{ }_{n}^{\mathrm{T}} \mathbf{M}_{n}}
$$

in which ${ }_{n}$ is the $n$th mode shape of the undamped system.

Once the modal damping ratios are computed, the response is computed by using techniques available for proportional systems (Equation (2)).

Note that the procedure described in this section is equivalent to neglecting off-diagonal terms of (Equation (17)). This implies that the coupling between the modal equations due to non-proportional nature of the damping matrix is ignored. Also note that this procedure is not new, as it has been proposed earlier [12-14] and has been adopted in the FEMA documents $[15,16]$. However, its applicability to asymmetric-plan systems with supplemental damping is explicitly explored for the first time in this study.

\section{EVALUATION OF SIMPLIFIED PROCEDURE}

The simplified procedure is implemented for a simple one-storey asymmetric-plan system with linear viscous dampers. This section first describes the system and ground motion considered in this study. The damping ratios from the simplified procedure are compared next with those obtained from complex eigenanalysis (Equation (12)). Also presented are the results for the parameter defined by Warburton and Soni [12]. Finally, the edge deformations from the simplified procedure are compared with those from the exact time-history analysis of the coupled system. 

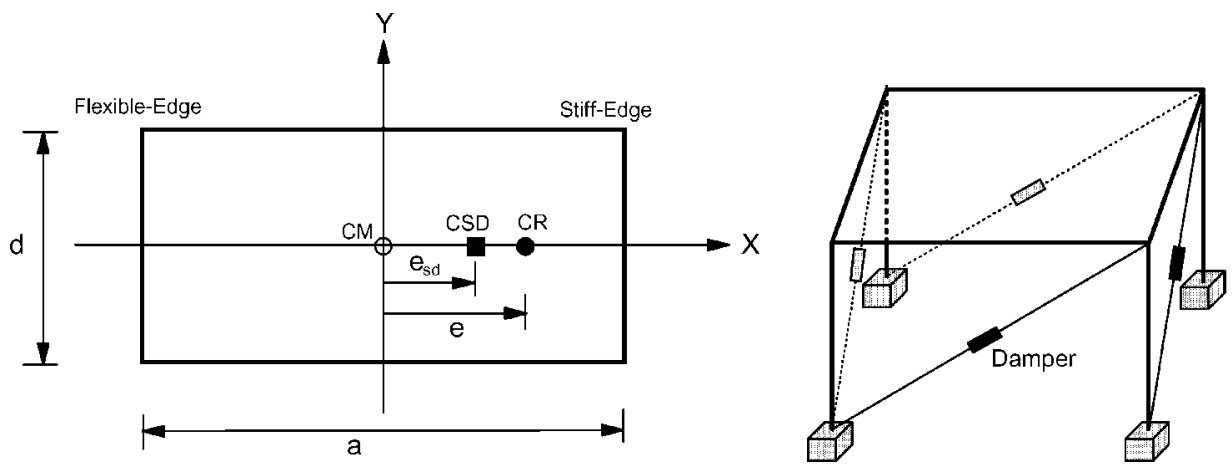

Figure 1. Idealized one-storey system.

\section{System and ground motion}

One-storey system. The system considered was the idealized one-storey building of Figure 1 consisting of a rigid deck supported by structural elements (wall, columns, moment-frames, braced-frames, etc.) in each of the two orthogonal directions, and included fluid viscous dampers incorporated into the bracing system. The mass properties of the system were assumed to be symmetric about both the $X$ - and $Y$-axis whereas the stiffness and the damper properties were considered to be symmetric only about the $X$-axis. The distance between the centre of mass (CM) and the centre of supplemental damping (CSD) is denoted by the supplemental damping eccentricity, $e_{\mathrm{sd}}$, whereas distance between the $\mathrm{CM}$ and the centre of rigidity $(\mathrm{CR})$ is defined by the stiffness eccentricities, $e$.

System matrices and parameters. The one-way symmetric system (Figure 1) has two DOF when subjected to ground motion along the $Y$-axis: translation along the $Y$-axis and rotation about a vertical axis. The displacement vector $\mathbf{u}$ for the system is defined by $\mathbf{u}^{\mathrm{T}}=u_{y} a u$ where $u_{y}$ is the horizontal displacement relative to the ground of the $\mathrm{CM}$ along the $Y$-axis, $u$ is the rotation of the deck about a vertical axis, and $a$ is the plan dimension of the system along the $X$-axis. The mass, stiffness, and damping matrices of the system with respect to the DOF $\mathbf{u}$ are then given in terms of the system parameters as

$$
\mathbf{M}=\left[\begin{array}{cc}
m & 0 \\
0 & \frac{1+^{2}}{12^{2}} m
\end{array}\right]
$$

where $m$ is the total deck mass and $=a / d$ is the aspect ratio of the deck,

$$
\mathbf{K}=m \omega_{y}^{2}\left[\begin{array}{cc}
1 & \bar{e} \\
\bar{e} & \bar{e}^{2}+\frac{1+{ }^{2}}{12^{2}} \Omega^{2}
\end{array}\right]
$$

in which $\omega_{y}$ is the undamped transverse vibration frequency of a corresponding uncoupled system defined as a system with coincidental $\mathrm{CM}$ and $\mathrm{CR}$ but with relative location and stiffness of all resisting elements identical to those in the asymmetric-plan system, $\Omega$ is ratio 
of the torsional and transverse frequencies of the corresponding uncoupled system, and $\bar{e}=e / a$. The damping matrix of the system can be formed from Equations (18) and (19) with the damping matrix due to supplemental dampers $\mathbf{C}_{\mathrm{sd}}$ given as

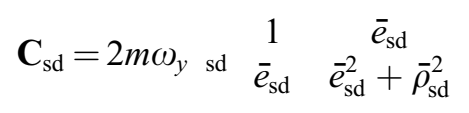

in which $s d_{\text {d }}$ is the supplemental damping ratio, $\bar{e}_{\mathrm{sd}}=e_{\mathrm{sd}} / a$ is the normalized supplemental damping eccentricity, and $\bar{\rho}_{\mathrm{sd}}=\rho_{\mathrm{sd}} / a$ is the normalized supplemental damping radius of gyration. Detailed descriptions of various system parameters and derivations of the system matrices are available elsewhere [17].

Selected system and damping parameters. The following parameters related to the system were fixed in this investigation: $\Omega=1$ to represents systems with strong coupling between lateral and torsional motions in the elastic range; $\bar{e}=0.2$ which implies an eccentricity of 20 per cent of the plan dimension; $=2 ; a_{0}$ and $a_{1}$ in Equation (18) to achieve damping ratios in both vibration modes of the system without supplemental damping equal to 5 per cent. Among the parameters related to the supplemental damping and its plan-wise distribution, sd was fixed at 10 per cent. The other parameters were varied over a wide range of practical values. For example, $\bar{e}_{\text {sd }}$ was varied between the extreme values of -0.5 to 0.5 corresponding to all dampers concentrated at either the flexible edge or the stiff edge of the system; whereas three discrete values of $\bar{\rho}_{\mathrm{sd}}=0,0.2$, and 0.5 representing low, medium, and large spreads of the supplemental damping about the CSD were considered. For selected cases, discrete values of $\bar{e}_{\mathrm{sd}}=-0.2,0$, and 0.2 were also considered; $\bar{e}_{\mathrm{sd}}=-0.2$ represents a plan-wise distribution of damping such that the CSD is located at a distance equal to the structural eccentricity $\bar{e}$ but on the side of the CM opposite to the CR, $\bar{e}_{\mathrm{sd}}=0.2$ represents identical locations of the $\mathrm{CSD}$ and $\mathrm{CR}$, and $\bar{e}_{\mathrm{sd}}=0$ implies even distribution of the supplemental damping, i.e., identical locations of the CM and the CSD.

Ground motion. The ground motion considered is the North-South $\left(360^{\circ}\right)$ component recorded at the Sylmar County Hospital parking lot during the 1994 Northridge earthquake. The peak values of the ground acceleration, velocity, and displacement recorded at the site were $826.6,128.9$, and $32.55 \mathrm{~cm} / \mathrm{s}^{2}$, respectively. This ground motion was applied to the system to act in the $Y$-direction.

\section{Modal damping ratios}

The damping ratios in each of the two modes of vibration of the system are computed from two methods: the complex-valued eigenanalysis (Equation (13)), and the simplified procedure in which the damping ratio is based on the diagonal terms of the transformed damping matrix $\Phi^{\mathrm{T}} \mathbf{C} \Phi$ (Equation (20)) while its off-diagonal terms are neglected. Note that the damping ratio obtained form the first analysis is the apparent damping ratio the system will exhibit when vibrating in its corresponding (complex-valued) mode shape. This damping ratio is denoted as "exact" value in this paper. The damping ratio obtained from the second method obviously involves several simplifications and is denoted as "approximate" value. The closeness of the 


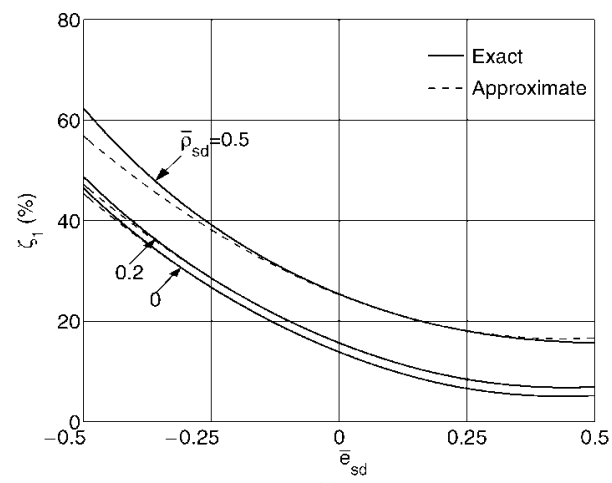

(a)

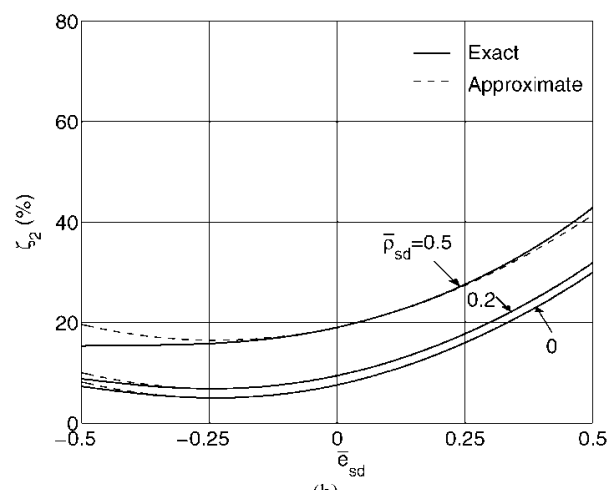

(b)

Figure 2. Comparison of apparent damping ratio from the state-space formulation with the damping ratios from the approximate procedure: (a) 1 and (b) 2 .

approximate damping ratio to be used in the simplified analysis to the exact damping ratio that is required to be used in the complex-values modal analysis will indicate the suitability of the simplified method for analysis of asymmetric-plan systems with supplemental damping.

The approximate and exact values of damping ratios are compared in Figure 2. These results show that over a wide range of parameters, the approximate damping ratio used in the simplified method is nearly the same as the exact damping ratio in the complex-valued modal analysis. This is especially true for low to moderate values of the $\bar{\rho}_{\text {sd }}(=0$ and 0.2$)$. For larger values of $\bar{\rho}_{\text {sd }}(=0.5)$, however, the two values may differ. These differences occur for extreme values of $\bar{e}_{\mathrm{sd}}$. For example, the approximate value is smaller for the first mode and larger for the second mode compared to the exact value in the neighbourhood of $\bar{e}_{\mathrm{sd}}=-0.5$; the trend reverses in the neighbourhood of $\bar{e}_{\mathrm{sd}}=0.5$. However, the differences are negligibly small.

Based on the results of Figure 2, it may be expected that the simplified method that uses the approximate value of damping ratio would lead to good estimate of the response for a wide range of system parameters. Noticeable errors may be expected only for systems with large values of $\bar{\rho}_{\mathrm{sd}}$ and extreme values of $\bar{e}_{\mathrm{sd}}$. 


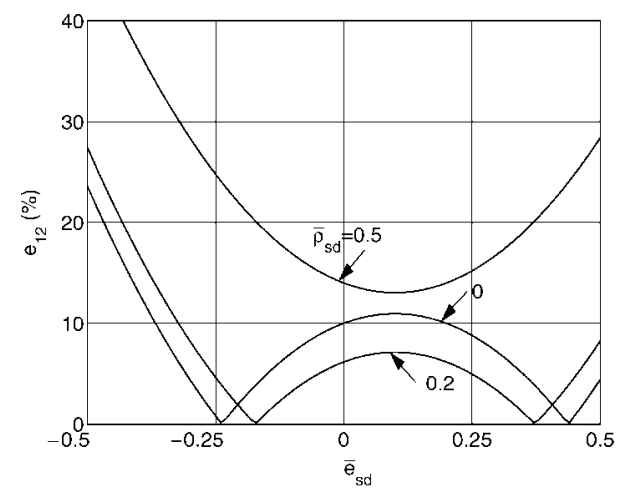

Figure 3. Error parameter defined by Warburton and Soni due to decoupling of damping matrix.

\section{Warburton and Soni's error parameter}

In order to assess the impact of neglecting the off-diagonal terms in the transformed damping matrix $\Phi^{\mathrm{T}} \mathbf{C} \Phi$, Warburton and Soni [12] proposed a parameter. This parameter can be expressed as follows for the two-degree-of-freedom system being considered in this study:

$$
e_{12}={ }_{1}^{\mathrm{T}} \mathbf{C} 2 \frac{\omega_{2}}{\omega_{2}^{2}-\omega_{1}^{2}}
$$

Variation of this parameter with $\bar{e}_{\mathrm{sd}}$ is presented in Figure 3 for three values of $\bar{\rho}_{\mathrm{sd}}=0,0.2$, and 0.5 . It is apparent from these results that the parameter is within 10 per cent over a wide range of $\bar{e}_{\mathrm{sd}}$ values for low to moderate values of $\bar{\rho}_{\mathrm{sd}}(=0$ and 0.2$)$; the parameter becomes large only in the neighbourhood of $\bar{e}_{\text {sd }}=-0.5$. For large value of $\bar{\rho}_{\text {sd }}(=0.5)$, however, the parameter exceeds 10 per cent for the entire range of $\bar{e}_{\text {sd }}$, with the value becoming particularly large in the neighbourhood of $\bar{e}_{\mathrm{sd}}=-0.5$ and 0.5 .

Warburton and Soni [12] recommended, based on studies of single- and multi-degree-offreedom systems, limiting the parameter to be no more than 5 per cent. For his value of the parameter, the maximum error in the response was shown to be no more than 10 per cent. For specific applications, higher values of the parameter may be acceptable depending on the desired accuracy of the results. It will be shown later in this study that response estimates from the simplified method within 15 per cent of the exact response may be obtained even for systems for which the parameter is up to 10 per cent.

\section{Edge deformations}

In order to evaluate the effects of neglecting off-diagonal terms in the transformed damping matrix, edge deformations of the system of Figure 1 due to the selected ground motion are computed by the following two methods and compared. The first method, denoted as the "exact" method, entails solving the coupled equations of motion (Equation (1)) in time domain. The second method, denoted as "approximate" method, involves solving the uncoupled modal equations with damping ratios obtained from the simplified procedure (Equation (20)); the modal equations are developed in terms of the mode shapes and frequencies of the undamped 


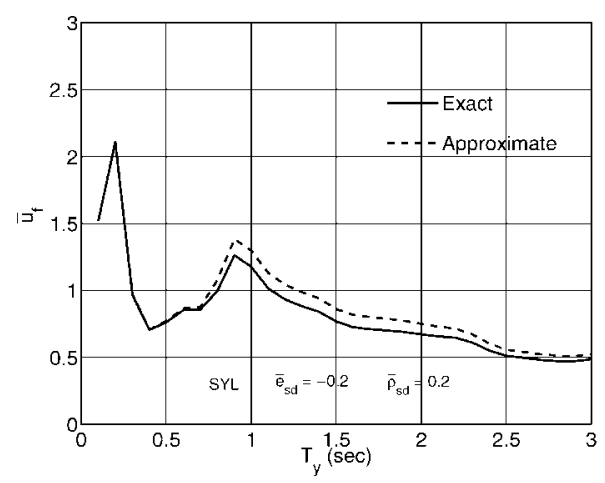

(a)

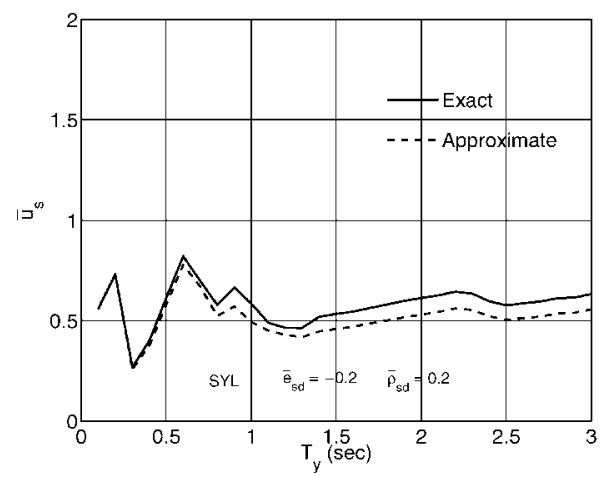

(b)

Figure 4. Comparison of normalized deformations from exact and approximate procedures: $\bar{e}_{\mathrm{sd}}=-0.2$;

(a) flexible-side element and (b) stiff-side element.

system. Since the response in the "exact" method is computed in the time domain, the modal equations in the "approximate" method are also solved in the time domain. In this study, instead of solving the modal equations, the response in the "approximate" method is obtained by solving the coupled equations (Equation (1)) with the damping matrix re-formed based on the modal damping ratios obtained from the simplified procedure (Equation (20)); note that for linear systems, the two procedures give the same response.

The normalized edge deformations, $\bar{u}_{\mathrm{s}}$ and $\bar{u}_{\mathrm{f}}$, are presented in Figures 4-6 for three different values of $\bar{e}_{\mathrm{sd}}=-0.2,0$ and 0.2 . The normalization is achieved by dividing the peak deformations of the stiff and flexible edge of an asymmetric-plan system, $u_{\mathrm{s}}$ and $u_{\mathrm{f}}$, respectively, by the peak deformation of the corresponding symmetric-plan system $u_{\mathrm{o}}$. Note that $u_{\mathrm{o}}$ is also the deformation at the two edges of the corresponding symmetric-plan system because such a system undergoes no torsional motion. The normalized edge deformations, $\bar{u}_{\mathrm{s}}$ and $\bar{u}_{\mathrm{f}}$, are indicative of the effects of plan asymmetry: a value of the normalized edge deformation more than one indicates that the edge deformation in the asymmetric-plan system is larger than the deformation of the same edge in the symmetric-plan system and vice versa.

The results presented in Figures $4-6$ indicate that for short-period systems, e.g., $T_{y}<0.5 \mathrm{sec}$, the two methods lead to nearly identical edge deformation, regardless of $\bar{e}_{\text {sd }}$ indicating that 


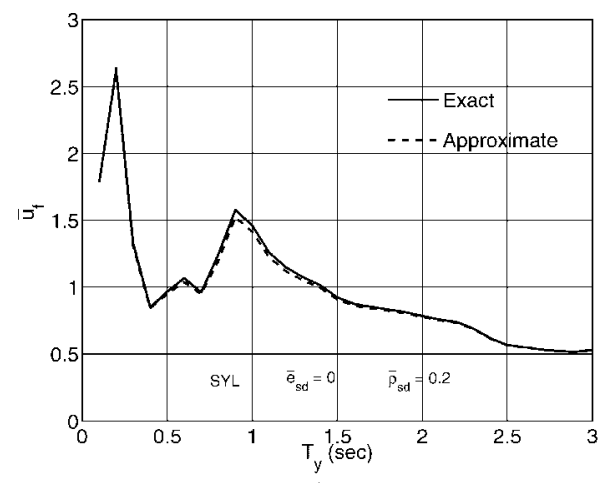

(a)

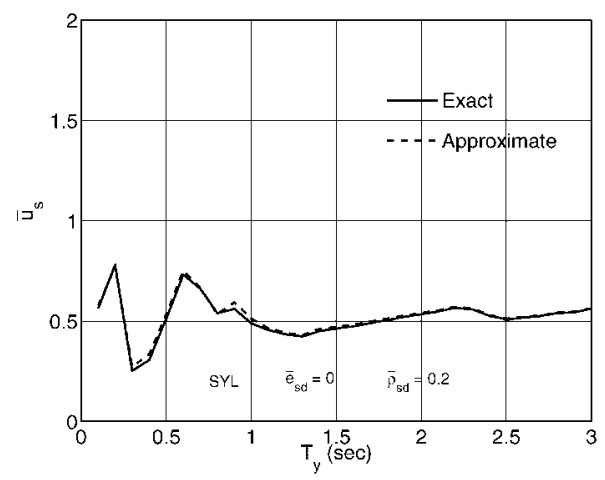

(b)

Figure 5. Comparison of normalized deformations from exact and approximate procedures: $\bar{e}_{\mathrm{sd}}=0$;

(a) flexible-side element and (b) stiff-side element.

the "approximate" method may be used without introducing any errors for the short period systems. This trend is applicable to both edges. For the longer period systems, however, the "approximate" method gives higher deformations of the flexible edge and smaller deformation of the stiff edge for $\bar{e}_{\mathrm{sd}}=-0.2$ (Figure 4), whereas the trend is reversed for $\bar{e}_{\mathrm{sd}}=0.2$ (Figure 6). However, the differences are small. For $\bar{e}_{\mathrm{sd}}=0$ (Figure 5), the "approximate" method gives deformations that are nearly the same as those from the "exact" method. These results indicate that the "approximate" method may be used for longer period systems provided some errors are acceptable.

In order to assess the level of difference between the results obtained from the "exact" and "approximate" procedures, the percentage errors were computed and are presented in Figure 7. It is apparent from this figure that the errors are, in general, less than 15 per cent. The errors are the smallest and nearly zero for $\bar{e}_{\mathrm{sd}}=0$. As expected based on the results of Figures 4-6, the errors are positive, indicating overestimation of the response, for the flexible edge (Figure 7a) and negative, indicating underestimation of the response, for the stiff edge (Figure $7 \mathrm{~b}$ ) of systems with $\bar{e}_{\mathrm{sd}}=-0.2$. These trends are reversed for systems with $\bar{e}_{\mathrm{sd}}=0.2$. 


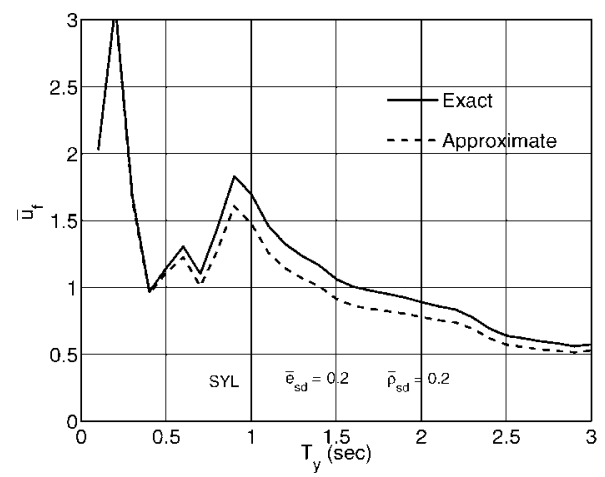

(a)

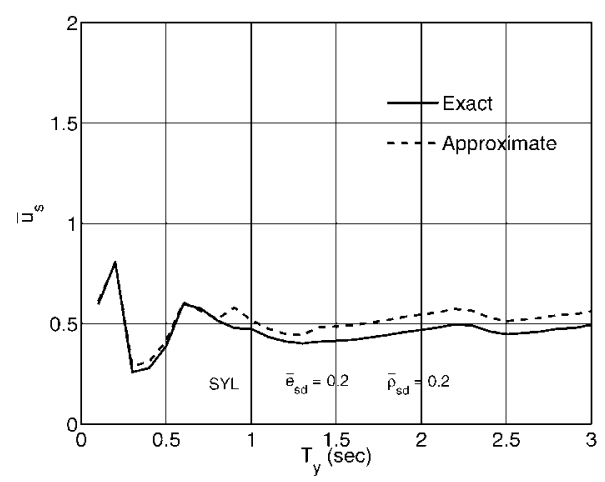

(b)

Figure 6. Comparison of normalized deformations from exact and approximate procedures: $\bar{e}_{\mathrm{sd}}=0.2$;

(a) flexible-side element and (b) stiff-side element.

The results presented in Figure 7 are for $\bar{\rho}_{\text {sd }}=0.2$. It would be useful to evaluate how the error trends differ for smaller or larger values of $\bar{\rho}_{\mathrm{sd}}$. Therefore, edge deformations were computed for systems with two other values $\bar{\rho}_{\text {sd }}=0$ and 0.5 and are presented in Figures 8 and 9 , respectively. These results show that the trends and level of errors for $\bar{\rho}_{\mathrm{sd}}=0$ are, in general, similar to those observed previously for $\bar{\rho}_{\mathrm{sd}}=0.2$. For $\bar{\rho}_{\mathrm{sd}}=0.5$, however, the errors tend to be slightly larger, with values for some cases exceeding 25 per cent, compared to those for $\bar{\rho}_{\mathrm{sd}}=0.2$. This observation is consistent with the earlier observation based on Warburton and Soni's parameter where the error parameter was found to be large for $\bar{\rho}_{\mathrm{sd}}=0.5$.

It is useful to point out that flexible edge in asymmetric-plan systems, in general, undergoes larger deformations during earthquake ground motions compared to the stiff edge, which is also apparent from the results presented in Figures $4-6$ where $\bar{u}_{\mathrm{f}}$ is, in general, much larger than $\bar{u}_{\mathrm{s}}$. Therefore, the plan-wise distribution of damping is selected to reduce deformations of the flexible edge. As observed in several earlier studies [6, 17, 18], and apparent from Figure 4, this objective is achieved by distributing supplemental damping in the system's plan such that $\bar{e}_{\mathrm{sd}}=-0.2$, i.e., the CSD is located at a distance equal to $\bar{e}$ from the CM but on the side opposite to the CR. For such a configuration, the "approximate" procedure gives deformation of the flexible edge to be slightly larger than that obtained from the "exact" procedure (Figures 4-6), 


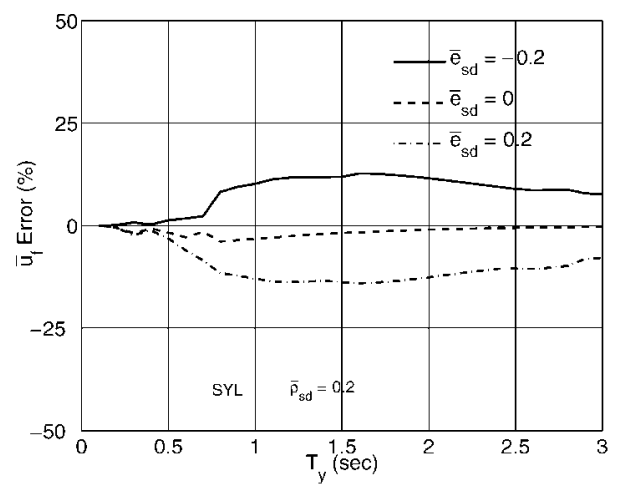

(a)

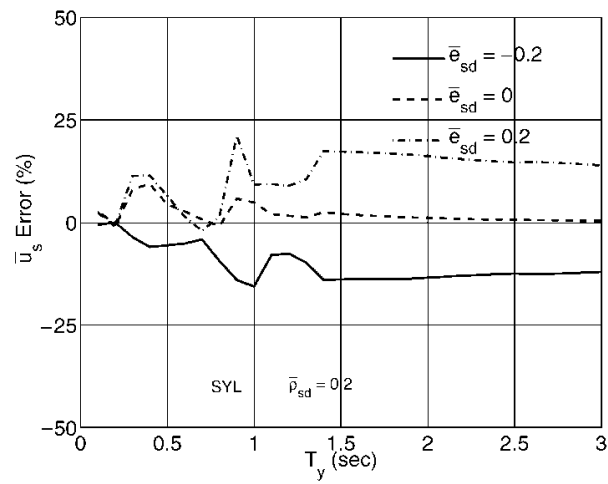

(h)

Figure 7. Errors in normalized deformations from approximate procedure: $\bar{\rho}_{\mathrm{sd}}=0.2$; (a) flexible-side element and (b) stiff-side element.

and the error rarely exceeds 15 per cent (Figures $7 \mathrm{a}-9 \mathrm{a}$ ). This indicates that the approximate procedure gives conservative results and the level of conservatism is not excessive.

If deformation of the stiff edge is of concern, as may be the case if brittle elements located on the stiff side are to be protected, the plan-wise distribution of supplemental damping should be selected to give $\bar{e}_{\mathrm{sd}}=0.2$, i.e., identical locations of the CSD and the CR. For such a configuration, the "approximate" procedure once again gives conservative estimate of the stiff-edge deformation (Figures $7 \mathrm{~b}-9 \mathrm{~b}$ ), although the level of conservatism may be slightly higher than that for the flexible edge.

The results presented so far are for a selected few values of $\bar{e}_{\mathrm{sd}}$. It would be useful to examine the variation of errors over a much wider range of $\bar{e}_{\mathrm{sd}}$ values. For this purpose, responses of systems with fixed period $\left(T_{y}=1 \mathrm{sec}\right)$ were computed for several values of $\bar{e}_{\text {sd }}$ ranging from -0.5 to 0.5 . The errors in response obtained from the "approximate" procedure are presented in Figure 10. These results show that errors are minimal for values of $\bar{e}_{\mathrm{sd}}$ in the neighbourhood of zero, i.e., for symmetric plan-wise distribution of supplemental damping. As $\bar{e}_{\mathrm{sd}}$ takes on increasing larger negative values, i.e., the CSD moves closer to the flexible edge, the errors in deformation of both edges increases. For the flexible edge, the error tends 


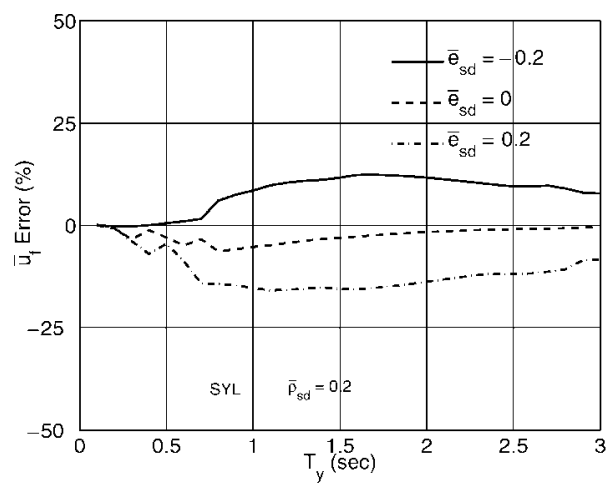

(a)

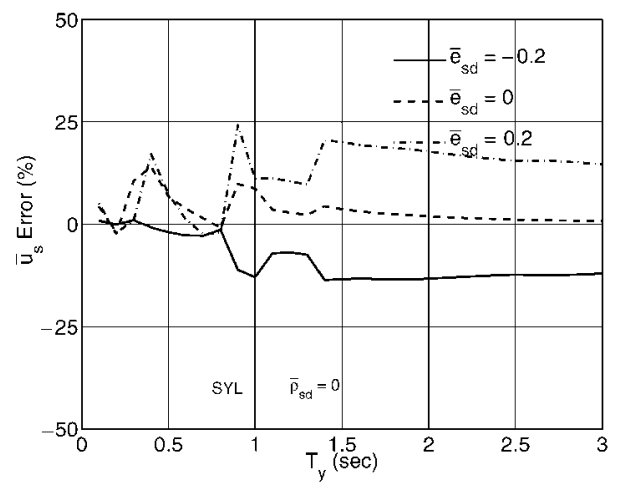

(b)

Figure 8. Errors in normalized deformations from approximate procedure: $\bar{\rho}_{\mathrm{sd}}=0$; (a) flexible-side element and (b) stiff-side element.

to be positive, implying the deformations from the "approximate" procedure are larger than those from the "exact" procedure. For the stiff edge, on the other hand, the error tends to be negative, implying that the deformations from the "approximate" procedure are smaller than those from the "exact" procedure. As $\bar{e}_{\text {sd }}$ approaches 0.5 , i.e., the CSD moves closer to the stiff edge, the trends in errors are reversed.

It is also apparent from these results that the error become excessively large for extreme values of $\bar{e}_{\mathrm{sd}}$ as CSD approaches the flexible edge, i.e., $\bar{e}_{\mathrm{sd}}$ approaches -0.5 . This is consistent with the previous observations based on the Warburton and Soni's [12] error parameter (Figure 3 ), which also becomes very large as $\bar{e}_{\text {sd }}$ approaches -0.5 .

For the selected system, the errors are within 15 per cent for values of $\bar{e}_{\text {sd }}$ approximately between -0.2 and 0.2 . Since the structural eccentricity for the selected system is $\bar{e}=0.2$, this implies that the "approximate" procedure can be used to reasonably and conservatively estimate the response of asymmetric-plan systems with supplemental damping for system with $-\bar{e} \leqslant \bar{e}_{\mathrm{sd}} \leqslant \bar{e}$, a range that has been suggested previously to give near optimal reduction in the edge deformations. In this range, the errors for systems with large plan-wise spread of supplemental damping $\left(\bar{\rho}_{\mathrm{sd}}=0.5\right)$ are within acceptable limits of say 15 per cent. 


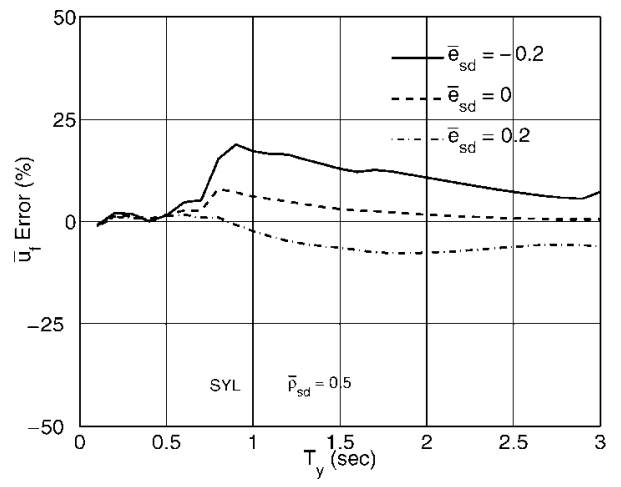

(a)

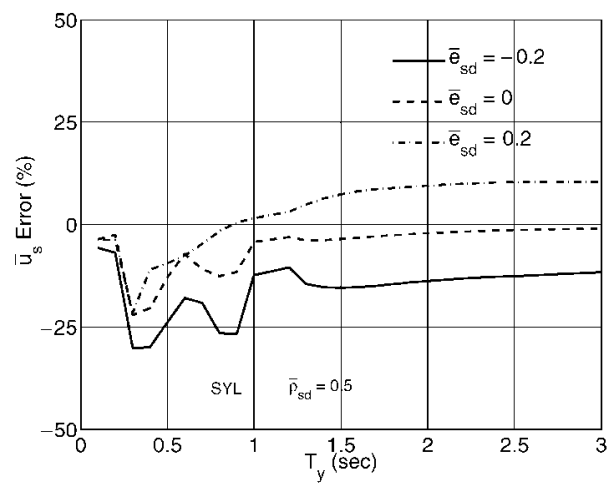

(b)

Figure 9. Errors in normalized deformations from approximate procedure: $\bar{\rho}_{\text {sd }}=0.5 ;$ (a) flexible-side element and (b) stiff-side element.

\section{CONCLUSIONS}

This study investigated the effects of neglecting the off-diagonal terms of the transformed damping matrix on the seismic response of asymmetric systems with supplemental damping, a class of systems which generally fall in the category of non-proportionally damped systems. Furthermore, it investigated the range of system parameters for which this simplification can be used without introducing significant errors in the response. For this purpose, damping properties, Warburton and Soni's parameter, and seismic response of one-storey asymmetric-plan system responding in the elastic range are examined for a wide range of system parameters. This investigation has led to the following conclusions:

1. Over a wide range of parameters, the "approximate" damping ratio obtained by neglecting the off-diagonal terms of the transformed damping matrix is nearly the same as the "exact" (or apparent) damping ratio in the complex-valued modal analysis indicating that the "approximate" value of damping ratio is suitable for use in the simplified method.

2. The error parameter, defined by Warburton and Soni [12] is within 10 per cent over a wide range of $\bar{e}_{\mathrm{sd}}$ values for low to moderate values of $\bar{\rho}_{\mathrm{sd}}(=0$ and 0.2$)$ but becomes 


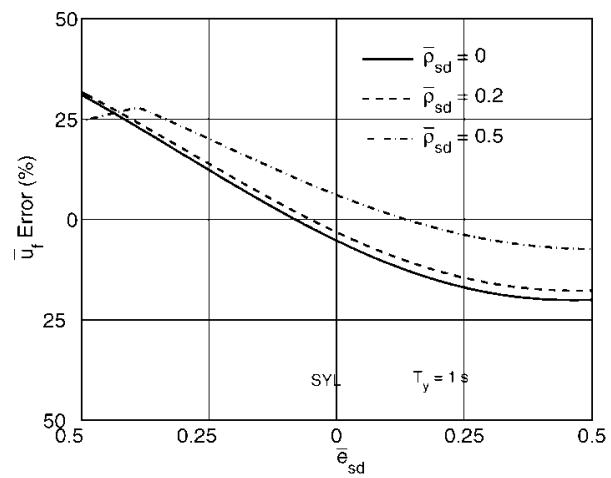

(a)

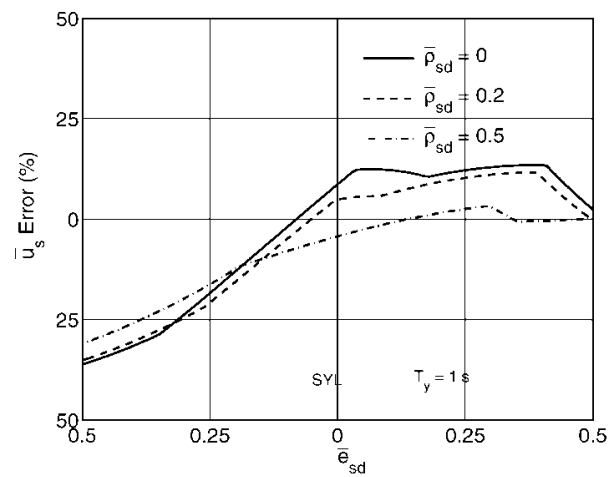

(b)

Figure 10. Errors in normalized deformations from approximate procedure: $T_{y}=1 \mathrm{~s}$; (a) flexible-side element and (b) stiff-side element.

excessive for values of $\bar{e}_{\mathrm{sd}}$ close to -0.5 . This indicates that the simplified procedure should not be used for asymmetric-plan systems with large damping eccentricity.

3. The simplified method may be used without introducing significant errors for the short period systems for the entire range of system parameters. For longer period systems, slightly larger errors should be expected.

4. The simplified method gives conservative estimate of the deformation at the flexible edge, which is most often the critical edge in asymmetric-plan systems.

5. The simplified method conservatively estimates the response of asymmetric-plan systems with supplemental damping with $-\bar{e} \leqslant \bar{e}_{\text {sd }} \leqslant \bar{e}$, a range that has been suggested previously to give the near optimal reduction in the edge deformations. In this range, the errors in edge deformations are within 15 per cent.

\section{ACKNOWLEDGEMENTS}

This research investigation is funded by the National Science Foundation under Grant CMS-9812414. This financial support is gratefully acknowledged. 


\section{REFERENCES}

1. Inman DJ. Engineering Vibrations. Prentice Hall: Englewood Cliffs, NJ, 1996.

2. Chopra AK. Dynamics of Structures: Theory and Applications to Earthquake Engineering. Prentice Hall: Upper Saddle River, NJ, 1995.

3. Clough RW, Mojtahedi S. Earthquake response analysis considering non-proportional damping. Earthquake Engineering and Structural Dynamics 1976; 4(5):489-496.

4. Igusa T, Der Kiureghian A, Sackman JL. Modal decomposition method for stationary response of non-classically damped systems. Earthquake Engineering and Structural Dynamics 1984; 12(1):121-136.

5. Veletsos AS, Ventura CE. Modal analysis of non-classically damped linear systems. Earthquake Engineering and Structural Dynamics 1986; 14(2):217-243.

6. Goel RK. Seismic behaviour of asymmetric buildings with supplemental damping. Earthquake Engineering and Structural Dynamics 2000; 29(3):461-480.

7. Claret AM, Venancio-Filho F. A modal superposition pseudo-force method for dynamic analysis of structural systems with non-proportional damping. Earthquake Engineering and Structural Dynamics 1991; 20(4): 303-315.

8. Ibrahimbegovic A, Chen HC, Wilson EL, Taylor RL. Ritz method for dynamic analysis of large discrete linear systems with non-proportional damping. Earthquake Engineering and Structural Dynamics 1990; 19(6): $877-889$.

9. Ibrahimbegovic A, Wilson EL. Simple numerical algorithms for the mode superposition analysis of linear structural systems with non-proportional damping. Computers \& Structures 1989; 33(2):523-531.

10. Jangid RS, Datta TK. Spectral analysis of systems with non-classical damping using classical mode superposition technique. Earthquake Engineering and Structural Dynamics 1993; 22(8):723-735.

11. Roesset JW, RV, Dobry R. Modal analysis for structures with foundation interaction. Journal of Structural Division ASCE 1973; 99(ST3):399-415.

12. Warburton GB, Soni SR. Error in response calculations for non-classically damped structures. Earthquake Engineering and Structural Dynamics 1977; 5(4):365-376.

13. Duncan PE, Taylor RE. A note on the dynamic analysis of non-proportionally damped systems. Earthquake Engineering and Structural Dynamics 1979; 7(1):99-105.

14. Sinha R, Igusa T. CQC and SRSS methods for non-classically damped structures. Earthquake Engineering and Structural Dynamics 1995; 24(4):615-619.

15. FEMA. NEHRP guidelines for the seismic rehabilitation of buildings. FEMA-273. Federal Emergency Management Agency, Washington, D.C., 1997.

16. FEMA. NEHRP Commentary on the guidelines for the seismic rehabilitation of buildings. FEMA-274. Federal Emergency Management Agency, Washington, D.C., 1997.

17. Goel RK. Effects of supplemental viscous damping on seismic response of asymmetric-plan systems. Earthquake Engineering and Structural Dynamics 1998; 27(2):125-141.

18. Goel RK, Booker CA. Effects of supplemental viscous damping on inelastic seismic response of asymmetric systems. Earthquake Engineering and Structural Dynamics 2001; 30(3):411-430. 